\title{
Avaliação comparativa entre custos dos processos MBBR/IFAS e lodo ativado para o tratamento de esgoto sanitário
}

\author{
Comparative evaluation between the costs of MBBR/IFAS and activated \\ sludge processes for sewage treatment
}

\section{Daniel Vieira Minegatti de Oliveira | Isaac Volschan Junior Roque Passos Piveli*}

Data de entrada: 30/05/2012 | Data de aprovação: 05/03/2013

Resumo

O processo MBBR/IFAS, Moving Bed Biofilm Reactor/Integrated Fixed Film Activated Sludge, é uma tecnologia recente para o tratamento de esgoto que incorpora tanto biomassa em suspensão como aderida. Seus parâmetros de controle são os mesmos aplicados ao processo de lodo ativado, observadas algumas especificidades. Como objetivo principal deste trabalho, apresenta-se uma análise comparativa entre os custos de implantação de uma unidade IFAS e de lodo ativado operando sob alta taxa, na faixa convencional e com aeração prolongada, para populações de 50.000 e 500.000 habitantes. Os resultados mostraram que, sob as hipóteses assumidas para a implantação dos reatores, o custo do processo de lodo ativado foi de $36 \%$ até $100 \%$ do custo do processo IFAS, dependendo da carga orgânica aplicada, do preço do terreno ou do custo do meio suporte.

Palavras-chave: Avaliação econômica, lodos ativados, IFAS, tratamento de esgotos.

\section{Abstract}

The Moving Bed Biofilm Reactor/Integrated Fixed Film Activated Sludge (MBBR/IFAS) process is a recent development technology that incorporates the characteristics of processes with biomass growth in suspension and attached biomass (biofilm). The control parameters are practically the same applied to the activated sludge process, saved some specifics. The principal aim this paper is presents a comparative analysis between the implantation costs of a new IFAS, high-rate, conventional and extended aeration activated sludge plant for a population of 50,000 and 500,000 inhabitants. The results indicated that according to the hypotheses assumed for the implantation of the reactors, the activated sludge process was of 36\% to 100\% more economical than the IFAS, depending on the organic loading rate, of the land price or of the carriers costs.

Key-words: Activated sludge, economic evaluation, integrated fixed film activated sludge, wastewater treatment

Daniel Vieira Minegatti de Oliveira*

Doutorando do Departamento de Engenharia Hidráulica e Sanitária da Escola Politécnica - USP. Mestre em Tecnologia de Saneamento Ambiental e Recursos Hídricos pela COPPE/UFRJ. Engenheiro Ambiental pela UFV.

Isaac Volschan Junior

D.Sc., Prof. Adjunto do Departamento de Recursos Hídricos e Meio Ambiente da Escola Politécnica - UFRJ.

Roque Passos Piveli

D.Sc., Professor Associado do Departamento de Engenharia Hidráulica e Ambiental da Escola Politécnica - USP.

*Endereço para correspondência: Universidade de São Paulo, Escola Politécnica, Departamento de Engenharia Hidráulica e Sanitária. Av. Prof. Almeida Prado, 271 - Butantã - São Paulo - SP - Brasil, CEP: 05508-900.

Tel: $+55(21) 7933-1515$

E-mail: daniel.minegatti@gmail.com 


\section{Introdução}

O processo de lodo ativado para o tratamento de efluentes é o mais aplicado no mundo e em 2014 completará um século de existência. Seu princípio de funcionamento se baseia no cultivo de biomassa ativa em suspensão em um tanque de aeração, que promove a floculação e a decomposição biológica da matéria coloidal do esgoto. Em seguida, esta biomassa é separada nos decantadores secundários e retorna para o processo, sendo o lodo excedente descartado do processo para a garantia de uma concentração celular aproximadamente constante no reator biológico.

O processo Integrated Fixed Film Activated Sludge (IFAS) constitui uma tecnologia recente que vem ganhando mercado e aplicação para o tratamento de efluentes. Pode ser traduzida como reator biológico com leito móvel, que pode ser adaptada ao processo de lodo ativado, de forma que além da biomassa em suspensão, tem-se a contribuição adicional de biomassa aderida. Esta tecnologia foi introduzida pelas autoridades responsáveis pelo controle de poluição da Noruega. A empresa norueguesa Kaldnes Miljoteknologi A/S, em parceria com a Universidade da Noruega, iniciou o desenvolvimento dos processos MBBR/IFAS, objetivando principalmente a ampliação da capacidade de tratamento de diversas ETE de pequeno porte existentes no país, sem ampliação das dimensões dos reatores biológicos (ØDEGAARD et al., 1994).

A principal diferença entre os processos MBBR e IFAS é a recirculação de lodo que acontece apenas no IFAS, da mesma forma em que se aplica no processo de lodo ativado. No interior do tanque de aeração dos processos MBBR/IFAS, o material de enchimento (pequenos anéis plásticos) é mantido em contínuo movimento pela mistura promovida pelo sistema de aeração, apresentando elevada mobilidade e, consequentemente, exposição e contato com a massa líquida. Trata-se assim de um reator biológico híbrido, no qual os micro-organismos são mantidos em suspensão no meio como também aderidos ao meio suporte.

A justificativa para a introdução de meio suporte no reator biológico é a disponibilização de área superficial para o crescimento de biomassa e elevação do tempo de residência celular. A formação de biomassa aderida, em adição a suspensão, permite o aumento da capacidade de decomposição da matéria orgânica carbonácea e da conversão de compostos nitrogenados, quando comparadas às que ocorrem no processo de lodo ativado. Nesse sen- tido, o processo demanda menores volumes para os reatores biológicos, reduzindo assim o custo da obra civil, constituindo alternativa atrativa para o aumento da capacidade de uma ETE, principalmente quando se tem maior restrição de área.

É importante ressaltar que em processo com biofilmes têm como característica apresentar estabilidade mesmo quando ocorrem variações nas condições operacionais, como sobrecargas hidráulicas, orgânicas e alterações na composição do esgoto afluente. Uma vez que as culturas estejam plenamente estabelecidas no meio suporte móvel, oferecem robustez ao sistema, podendo operar sob condições em que o lodo em suspensão apenas não seria capaz. A nitrificação do esgoto também é beneficiada pela estabilidade do processo, à medida que populações significativas de bactérias nitrificantes estabelecem-se no biofilme, de forma que a oxidação da amônia ocorre com idades do lodo baixas, quando referenciadas apenas à biomassa em suspensão (Rusten et al., 1995).

O excesso de biofilme se desprende naturalmente do suporte, cedendo superfície para a ocupação de outros micro-organismos e os fragmentos liberados são facilmente separados da fase líquida em etapa seguinte, por serem mais compactos e consequentemente sedimentarem mais facilmente que os flocos do lodo convencional.

Dentre as principais vantagens desta tecnologia tem-se a obtenção de sistemas compactos, resistentes aos picos de cargas orgânicas e hidráulicas e às variações de $\mathrm{pH}$ e temperatura; bem como flexíveis pela possibilidade de variação da taxa de recirculação da biomassa suspensa para o melhor ajuste das condições operacionais (ØDEGAARD et al., 1994).

As variáveis de controle são as mesmas aplicadas ao processo de lodo ativado, guardada a especificidade de que a concentração de sólidos biológicos no tanque de aeração é aumentada pela formação de biomassa aderida. Esta deverá ser quantificada e somada à biomassa em suspensão para controle da relação alimento/micro-organismos. Além disso, no controle de reatores com biomassa aderida, costuma-se relacionar a carga orgânica aplicada com a área superficial total do meio suporte, ou carga orgânica superficial (COS), expressa em $\mathrm{gDBO} / \mathrm{m}^{2} . \mathrm{d}$. Os limites para este parâmetro são definidos pelas necessidades de remover apenas matéria orgânica ou também obter a nitrificação do esgoto.

O material utilizado para a fabricação do meio suporte é usualmente polietileno ou polipropileno, cuja densidade varia entre 0,95 a $0,99 \mathrm{~g} / \mathrm{cm}^{3}$; di- 

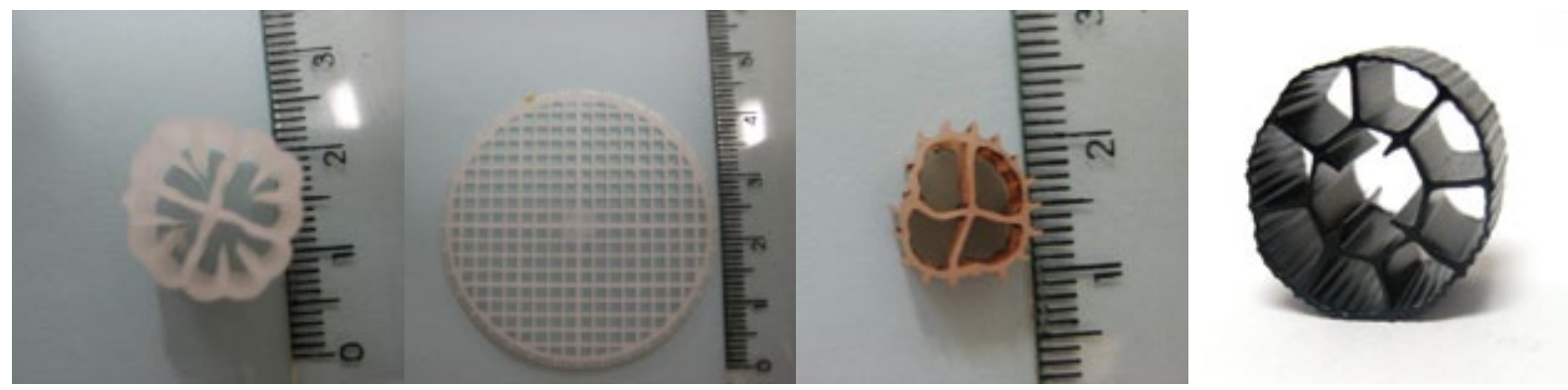

Figura 1 - Meios suporte empregados no processo MBBR/IFAS

âmetro entre 10 e $45 \mathrm{~mm}$ e largura entre 7 e 30 mm têm sido empregados. Apresentam-se, na Figura 1, alguns tipos de meio suporte empregados atualmente no processo MBBR/IFAS.

As diferentes configurações de meio suporte resultam em diferentes áreas de contato, as quais podem apresentar maior ou menor potencial para aderência de biomassa em função do arranjo e desenho geométrico da peça. Neste sentido, o meio suporte pode ser caracterizado tanto em função da superfície total disponível, assim como em função de uma parcela desta, a qual corresponde à área em que realmente ocorre a efetiva aderência de biomassa. As áreas superficiais específicas dos anéis existentes no mercado atualmente variam entre 350 e $900 \mathrm{~m}^{2} / \mathrm{m}^{3}$. (MINEGATTI, 2008).

$\mathrm{O}$ volume de meio suporte normalmente inserido no reator é de $20 \%$ a $70 \%$ do volume do tanque. Para densidades inferiores a $20 \%$, há uma perda na eficiência de transferência de oxigênio pela falta de material para efetivar a redução dos tamanhos das bolhas de ar introduzidas pelo equipamento de aeração. Para densidades superiores a $70 \%$, não se obtém boa mistura da mídia plástica e o custo da energia para fornecimento do oxigênio demandado pela elevada quantidade de biomassa torna-se elevado (RUSTEN et al., 1998).

Em relação ao sistema de aeração empregado no processo IFAS, deve-se levar em conta o fornecimento de oxigênio para satisfazer a demanda da biomassa adicional para a remoção de matéria orgânica e para a nitrificação do esgoto, além da necessidade de manutenção do meio suporte em movimento. Para isso, utilizam-se sistemas de bolhas grossas ou médias, pois o meio suporte favorece a absorção de oxigênio por quebra destas bolhas em bolhas finas e garante maior tempo de retenção das bolhas no meio, aumentando a efi- ciência na transferência de oxigênio. Ressalta-se que, em casos de conversão de lodo ativado em IFAS, a quantidade adicional de oxigênio requerida pelo aumento da carga orgânica aplicada, pode ser introduzida pelo sistema de aeração por bolhas finas pré-existente, instalando-se novos sopradores de ar.

Os resultados das pesquisas têm demonstrado que o processo MBBR/IFAS exige a manutenção de concentrações de oxigênio dissolvido nos tanques de aeração mais elevadas que no processo de lodo ativado, quando se deseja a nitrificação do esgoto. Nos estudos desenvolvidos por Rusten et al., (1995) foi obtida a correlação entre taxa de nitrificação e concentração de oxigênio dissolvido no tanque, reproduzida na Figura 2.

Com base neste gráfico, inferiu-se que a camada líquida à superfície do biofilme formado nos corpos móveis é limitante para a transferência de oxigênio. Como o oxigênio dissolvido é consumido para degradação de matéria orgânica por micro-organismos heterotróficos, que se posicio-

Figura 2 - Relação entre taxa de nitrificação e concentração de OD no processo MBBR/IFAS (Adaptado RUSTEN et al., 1995)

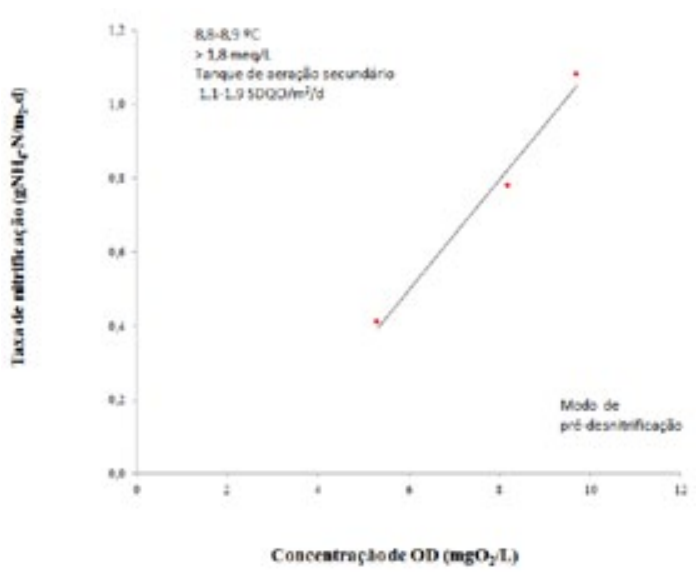


nam na parte externa do biofilme, para obtenção da nitrificação o nível de oxigênio dissolvido deve ser elevado para que haja penetração nas camadas internas, região em que predominam as bactérias nitrificantes. Nesta Figura 2, observa-se uma relação linear entre a concentração de oxigênio dissolvido e a taxa de nitrificação. A reta deve interceptar o eixo das abscissas entre 2,5 e 3,0 mgO $/$ L, faixa considerada mínima para ocorrência da nitrificação. Essa constatação é muito relevante, uma vez que o consumo de energia elétrica para aeração do sistema deverá ser substancialmente maior relativamente ao processo de lodo ativado apenas com biomassa em suspensão, em que a concentração de oxigênio dissolvido é mantida entre 1,0 e 2,0 $\mathrm{mgO}_{2} / \mathrm{L}$.

A Revisão da Norma Técnica NBR 12.209 da Associação Brasileira de Normas Técnicas (ABNT) recomenda manter no reator, para o caso de remoção de nitrogênio, uma concentração de OD no tanque de aeração de 3,0 mg/L. Isto faz com que o consumo de oxigênio no processo MBBR/ IFAS seja maior do que no lodo ativado, em que se mantém OD abaixo de 2,0 mg/L, com importante consequência sobre os custos operacionais.

Portanto, teoricamnte, a utilização do processo MBBR/IFAS é vantajosa economicamente em relação ao processo de lodo ativado, pelo fato de permitir a implantação de unidades de menores dimensões. No entanto, o custo elevado do meio suporte e o maior consumo de energia elétrica, compõem fatores desfavoráveis ao recurso da adição de corpos móveis nos tanques de aeração do processo de lodo ativado, quando se deseja aumentar sua capacidade.

O presente trabalho tem como principal objetivo avaliar a viabilidade econômica da aplicação do processo IFAS, comparando-o com o processo de lodo ativado operando como alta taxa, na faixa convencional e com aeração prolongada para o atendi- mento de populações equivalentes de 50.000 (IFAS $\mathrm{x}$ aeração prolongada), 500.000 habitantes (IFAS $x$ lodo ativado convencional) e 500.000 habitantes (IFAS x lodo ativado alta taxa). Foram levados em consideração os custos da obra de concreto armado, do sistema de aeração, do meio suporte e devidos ao consumo de energia elétrica. Foram levantados custos de terreno para a implantação do tratamento em cinco diferentes municípios das regiões metropolitanas de São Paulo e Campinas.

\section{Metodologia}

A avaliação econômica da implantação dos processos IFAS e lodo ativado, sob alta taxa, convencional e com aeração prolongada foi realizada a partir dos parâmetros tradicionais de dimensionamento encontrados na literatura especializada e também recomendados pela ABNT. As populações de projeto adotadas para este estudo foram 50.000 e 500.000 habitantes, considerando-se o consumo per capita de água de $200 \mathrm{~L} / \mathrm{hab} . d$, o coeficiente de retorno de 0,8 e contribuições per capita de 54 gDBO/hab.dia. Tem-se por resultado as vazões médias de esgoto de $8.000 \mathrm{~m}^{3} / \mathrm{d}$ e $80.000 \mathrm{~m}^{3} / \mathrm{d}$, correspondentes às cargas de DBO de 2,7 ton/d e 27 ton/d, respectivamente.

Foram considerados 3 diferentes cenários para a comparação dos processos: (i) somente remoção de matéria orgânica (DBO), contemplando IFAS e lodo ativado de alta taxa; (ii) remoção de matéria orgânica (DBO) + nitrificação, considerando IFAS e lodo ativado convencional; (iii) remoção de matéria orgânica $(\mathrm{DBO})$ + nitrificação + estabilização do lodo, contemplando IFAS e lodo ativado com aeração prolongada.

Nos dimensionamentos efetuados, para servirem como base para a composição de custos, foram consideradas as premissas de cálculo apresentadas na Tabela 1.

Tabela 1- Condições estabelecidas para a realização dos dimensionamentos

\begin{tabular}{|c|c|c|c|c|}
\hline \multicolumn{2}{|c|}{ Condições } & Alta taxa & Convencional & Aeração Prolongada \\
\hline \multicolumn{2}{|c|}{ População (hab.) } & 500.000 & 500.000 & 50.000 \\
\hline \multicolumn{2}{|c|}{ Relação A/M (kgDBO/kgSSV.d) } & 0,6 & 0,4 & 0,15 \\
\hline \multicolumn{2}{|l|}{$\mathrm{SSV}_{\mathrm{TA}}(\mathrm{mg} / \mathrm{L})$} & 2,0 & 2,5 & 3,0 \\
\hline \multicolumn{2}{|c|}{$\mathrm{NEC} \mathrm{O}_{2}\left(\mathrm{kgO}_{2} / \mathrm{kgDBO}\right)$} & 1,5 & 2,0 & 2,5 \\
\hline \multirow{2}{*}{$\mathrm{OD}_{\mathrm{TA}}(\mathrm{mg} / \mathrm{L})$} & Sem meio suporte & 1,5 & 1,5 & 1,5 \\
\hline & Com meio suporte & 1,5 & 3,0 & 3,0 \\
\hline
\end{tabular}


As estimativas dos custos de implantação como o estrutural de fundações, lajes, ferragem, etc., foram obtidas com o auxílio do aplicativo SIENGE - Software para a Indústria da Construção, que utiliza um software ERP (Enterprise Resource Planning) específico para Construção Civil, para uso do qual primeiramente deve-se fornecer a quantidade total de suprimentos necessários (cimento, pedras britadas, ferragens, mão de obra, etc.), sendo que posteriormente o software faz uma varredura online no mercado e fornece assim todos os custos solicitados. Isto foi realizado para cada um dos dimensionamentos, levando-se em conta as diferentes populações e tecnologias.

Foram incorporados também os custos do meio suporte e dos sistemas de aeração (soprador de ar, difusores, tubulação, etc.). Foi considerado que o valor de cada $\mathrm{m}^{3}$ do meio suporte empregado no processo IFAS é de $\mathrm{R} \$ 2.000,00$, reproduzindo o preço médio praticado no Brasil. Ademais, os valores considerados no custo das áreas ocupadas pelas ETE foram obtidos por meio de levantamento de áreas isoladas dentro dos municípios, com potencial de uso para este fim.

Embora este trabalho seja restrito à avaliação dos custos de implantação dos sistemas de tratamento, também será feita consideração a respeito dos gastos com energia elétrica esperados para os processos, podendo as diferenças nos valores desta importante condição operacional influírem decisivamente na escolha do processo a ser utilizado.

Assim, o consumo de energia elétrica pode ser estimado de acordo com modelo proposto por COSTA \& SILVA, (2011) e representado na equação a seguir, considerando que os serviços de saneamento se enquadram no subgrupo de tensão A4, tarifa verde.

$$
F t=D f a t \times T d+C p \times T c p+C f p \times T c f p
$$

Onde: $\mathrm{Ft}$ = valor da fatura $(\mathrm{R} \$)$; Dfat = demanda faturável $(\mathrm{kW}) ; \mathrm{Td}=$ tarifa de demanda faturável $(\mathrm{R} \$ / \mathrm{kW}) ; \mathrm{Cp}=$ consumo de energia elétrica medido no mês - horário de ponta (kW.h); Tcp = tarifa de consumo no horário de ponta (R\$/kW.h);
Cfp = consumo medido no mês - horário fora de ponta $(\mathrm{kWh})$; Tcfp = tarifa de consumo no horário - fora de ponta (R\$/kW.h).

\section{Resultados e discussão}

Os dimensionamentos foram feitos com base na relação $\mathrm{A} / \mathrm{M}$, sendo que no caso do processo IFAS considerou-se a contribuição adicional da biomassa aderida no meio suporte móvel introduzido nos tanques de aeração. Para os dimensionamentos das alternativas com processos operando sob alta taxa e taxa convencional (população de 500.000 habitantes), considerou-se que a ETE possuirá decantadores primários reduzindo 30\% da DBO do esgoto previamente ao tratamento biológico.

Nos cenários estabelecidos neste trabalho, não foi considerada a existência de câmaras anóxicas de pré-desnitrificação. Assim, estes cenários reproduzem a situação de diversos sistemas de lodos ativados existentes no Brasil e que se encontram em fase de estudos para a ampliação de suas capacidades de atendimento.

Será mostrado apenas o dimensionamento efetuado para o processo IFAS considerando população de 500.000 habitantes operando sob alta taxa. Para as outras combinações serão apresentados apenas os resultados finais dos dimensionamentos, que foram efetuados segundo os mesmos procedimentos.

Dimensionamento do processo IFAS para o atendimento a 500.000 habitantes operando apenas para a remoção de matéria orgânica (alta taxa)

Dimensões do Reator Biológico

Valores considerados:

Área superficial específica do meio suporte: $600 \mathrm{~m}^{2} / \mathrm{m}^{3}$

Massa de SSV aderida ao meio suporte: $12,0 \mathrm{~g} / \mathrm{m}^{2}$

Volume de meio suporte: $50 \%$ do volume do reator

Sob tais premissas, obtém-se o volume do reator biológico de:

$$
\begin{aligned}
& \frac{A}{M}=\frac{Q \times D B O}{X_{\mathrm{v}} x V_{T A}} \Rightarrow 0,6= \\
& \frac{0,7 \times 27.000}{0,5 \times V_{T A} \times 0,012 \times 600+2,0 \times V_{T A}} \Rightarrow \\
& V_{T A}=5.625 \mathrm{~m}^{3}
\end{aligned}
$$


Considerando-se a profundidade útil de $6 \mathrm{~m}$, tem-se a seguinte área necessária de tanques de aeração:

$$
A=\frac{V o l}{h} \Rightarrow A=\frac{5625}{6,0}=938 m^{2}
$$

Necessidade de Oxigênio e Consumo de Energia

$$
N E C \times O_{2}=1,5 \times(0,7 \times 27.000)=28.350 \mathrm{~kg} / \mathrm{d}
$$

Eficiência do difusor em campo

Para a profundidade do líquido de $6,0 \mathrm{~m}$, será considerada a eficiência do difusor em condições de teste $\left(\eta_{0}\right)$ igual a 30\%.

Correção para as condições de campo:

$$
n=n_{0} \times \lambda
$$

Onde $\lambda$ é:

$$
\lambda=\alpha \times \frac{\left(\beta C_{S W}-C_{L}\right) \times 1,02^{T-20}}{9,17}
$$

considerando $\alpha=0,70, \beta=0,95, \mathrm{CSW}=7,7$ $\mathrm{mg} / \mathrm{L}$ para $\mathrm{T}=28^{\circ} \mathrm{C}$ (temperatura do líquido no verão), $\mathrm{CL}=1,5 \mathrm{mg} / \mathrm{L}$, obtém-se:

$$
\lambda=0,7 \times \frac{(0,95 \times 7,7-1,5) \times 1,02^{8}}{9,17}=0,52
$$

Assim, o valor da eficiência do difusor em campo $(\eta)$ igual a $\eta=0,52 \times 30 \%=15,6 \%$.

\section{Vazão de ar necessária}

Valores considerados:

Massa especifica do $\mathrm{Ar}=1,2 \mathrm{~kg} / \mathrm{m}^{3}$

Teor de $\mathrm{O}_{2}$ no $\mathrm{Ar}=0,232 \mathrm{~kg} \mathrm{O} / \mathrm{kg} \mathrm{Ar}$

Vazão dos sopradores de ar:

$$
\begin{gathered}
Q_{a r}=\frac{N E C \times O_{2}}{0,156 \times 1,2 \times 0,232}= \\
\frac{28.350}{0,1158 \times 1,2 \times 0,232}= \\
652.654 \mathrm{~m}^{3} / \text { dia }=453 \mathrm{~m}^{3} / \mathrm{min}
\end{gathered}
$$

Considerando-se o acréscimo de 40\% para atender às demandas de pico, a vazão necessária de ar será de $635 \mathrm{~m}^{3} / \mathrm{min}$.

\section{Potência Necessária}

Valores considerados:

Massa de $\operatorname{Ar}\left(\mathrm{M}_{\mathrm{Ar}}\right)=\left(\mathrm{Q}_{\mathrm{Ar}} \times\right.$ Massa especifica de Ar) $/ 60$ minutos $=12,69 \mathrm{~kg} / \mathrm{s}$

Constante do gás $(\mathrm{R})=8,31 \mathrm{~kJ} / \mathrm{k} \mathrm{mol}^{\circ} \mathrm{K}$

Temperatura absoluta de entrada $(\mathrm{To})=$ $28{ }^{\circ} \mathrm{C} \rightarrow \mathrm{K}=28{ }^{\circ} \mathrm{C}+273,15=301,15^{\circ} \mathrm{K}$

Eficiência do compressor $(\mathrm{E})=0,7$

Pressão absoluta de entrada $(\mathrm{Pe})=1 \mathrm{~atm}$

Pressão absoluta de saída $(\mathrm{Ps}) \approx 1,3 \mathrm{x}$ pro-

$$
\begin{aligned}
& \text { Pot }=\frac{M_{A \mathrm{r}} \times R \times T_{0}}{8,41 \times E}\left\{\left(\frac{P_{S}}{P_{e}}\right)^{0,283}-1\right\}= \\
& \frac{12,69 \times 8,31 \times 301,15}{8,41 \times 0,7}\left\{\left(\frac{1,75}{1}\right)^{0,283}-1\right\} \Rightarrow \\
& \text { Pot }=933 \mathrm{~kW}
\end{aligned}
$$


fundidade da lâmina d`água $=1,3 \times 6,0 \mathrm{~m}=$ 7,8 m.c. $\mathrm{a}=0,75 \mathrm{~atm} ;=>$ Ps deve ser $=1+0,75$ $=1,75 \mathrm{~atm}$

\section{Resultados dos demais dimensionamentos}

Na Tabela 2 apresenta-se um resumo dos principais resultados obtidos nos dimensionamentos efetuados. prolongada, respectivamente.

No entanto, para realização de uma análise mais criteriosa, deve-se contabilizar também o custo da área ocupada pela ETE. Em vista disso, foram identificados os preços de terreno em áreas periféricas em municípios da Região Metropolitana de São Paulo e de Campinas, conforme apresenta a Tabela 4. Incorporando os valores da Tabela 4,

\begin{tabular}{l|c|c|c|c}
\multirow{2}{*}{ Condição de Projeto } & \multicolumn{2}{|c|}{ IFAS } & \multicolumn{2}{c}{ Lodo Ativado } \\
\cline { 2 - 5 } & VU (m3) & Pot (kW) & VU (m3) & Pot (kW) \\
\hline $\begin{array}{l}\text { Alta taxa } \\
500.000 \text { habitantes }\end{array}$ & 5.625 & 933 & 15.750 & 933 \\
\hline $\begin{array}{l}\text { Convencional } \\
500.000 \text { habitantes }\end{array}$ & 7.746 & 1.672 & 18.900 & 1.253 \\
\hline $\begin{array}{l}\text { Aeração Prolongada } \\
\text { 50.000 habitantes }\end{array}$ & 2.951 & 298 & 6.000 & 221 \\
\hline
\end{tabular}

Tabela 2- Volumes úteis dos tanques de aeração e potências instaladas

Para estimativa de custos da construção civil, como mencionado, foi utilizado o aplicativo SIENGE; e os custos dos sistemas de aeração foram obtidos juntamente com as empresas fornecedoras. Na Tabela 3 apresentam-se as estimativas dos custos de implantação do tanque de aeração para cada uma das tecnologias e condições operacionais que foram avaliadas.

Nota-se que, o processo de lodo ativado, independente da sua modalidade, resultou sempre em menor custo de implantação dos tanques de aeração, principalmente devido ao elevado custo de aquisição do meio suporte para o processo IFAS, correspondendo a $78 \%, 80 \%$ e $79 \%$ do custo total das modalidades alta taxa, convencional e aeração apresentam-se nas Tabelas 5 e 6, os custos finais de implantação dos processos dimensionados para ambas as populações.

Salienta-se que se optou por estas regiões pelo fato de estarem em ampla expansão habitacional e que se inseriu a relação entre custo final de implantação do processo lodo ativado e o custo final do processo IFAS (R).

Observa-se que o custo de implantação dos tanques de aeração para o processo IFAS resultou mais elevado que para o lodo ativado, independentemente da população de projeto ou do valor do terreno. Pode-se observar também que as diferenças percentuais são reduzidas à medida que a taxa de aplicação de matéria orgânica é aumentada.

Tabela 3- Estimativa dos custos de implantação dos processos avaliados

\begin{tabular}{c|c|c|c|c|c}
\multirow{2}{*}{$\begin{array}{c}\text { População } \\
\text { (hab.) }\end{array}$} & Processos & \multicolumn{4}{|c}{ Custos Específicos (R\$) } \\
\cline { 2 - 6 } & Lonstrução Civil & Meio Suporte & Sistema de Aeração & Total \\
\hline \multirow{2}{*}{500.000} & Lodo ativado alta taxa & 2.847 .690 & - & 536.297 & 3.383 .987 \\
\cline { 2 - 6 } & IFAS & 1.056 .947 & 5.625 .000 & 536.297 & 7.218 .244 \\
\hline \multirow{2}{*}{500.000} & Lodo ativado convencional & 3.168 .366 & - & 762.538 & 3.930 .904 \\
\cline { 2 - 6 } & IFAS & 1.292 .964 & 7.746 .000 & 609.881 & 9.648 .845 \\
\hline \multirow{2}{*}{50.000} & Lodo ativado aeração prolongada & 950.056 & - & 274.430 & 1.224 .485 \\
\cline { 2 - 6 } & IFAS & 460.918 & 2.951 .000 & 303.290 & 3.715 .208
\end{tabular}




\begin{tabular}{|c|c|c|c|c|c|c|}
\hline \multirow{2}{*}{ Processos } & \multirow{2}{*}{ Área $\left(\mathrm{m}^{2}\right)$} & \multicolumn{5}{|c|}{ Cidades } \\
\hline & & Atibaia $^{(1)}$ & Vinhedo $^{(2)}$ & Jundiaí(3) & Valinhos ${ }^{(4)}$ & São Paulo(5) \\
\hline $\begin{array}{l}\text { Lodo ativado } \\
\text { alta taxa }\end{array}$ & 2625 & 315.000 & 420.000 & 472.500 & 551.250 & 1.128 .750 \\
\hline $\mathrm{IFAS}^{(6)}$ & 3150 & 112.320 & 149.760 & 168.480 & 196.560 & 402.480 \\
\hline $\begin{array}{l}\text { Lodo ativado } \\
\text { convencional }\end{array}$ & 1000 & 378.000 & 504.000 & 567.000 & 661.500 & 1.354 .500 \\
\hline $\mathrm{IFAS}^{(7)}$ & 938 & 154.920 & 206.560 & 232.380 & 271.110 & 555.130 \\
\hline $\begin{array}{l}\text { Lodo ativado } \\
\text { aeração prolongada }\end{array}$ & 1291 & 163.680 & 218.240 & 245.520 & 286.440 & 586.520 \\
\hline IFAS $(8)$ & 492 & 103.920 & 138.560 & 155.880 & 181.860 & 372.380 \\
\hline
\end{tabular}

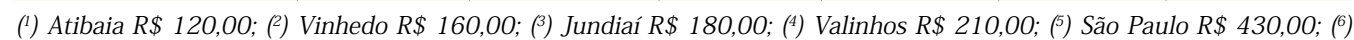
IFAS com A/M de 0,6 kgDBO/kgSSV.d; (7) IFAS com A/M de 0,4 kgDBO/kgSSV.d; ( () IFAS com A/M de 0,15 kgDBO/kgSSV.d

Tabela 4- Área requerida e o respectivo custo total para o tanque de aeração

Para a variante com aeração prolongada e população de 50.000 habitantes, o custo final de implantação do processo de lodo ativado resultou entre $36 \%$ e $44 \%$ do custo do processo IFAS. Com a população de projeto de 500.000 habitantes, para a faixa convencional o custo final de implantação do processo de lodo ativado resultou de $44 \%$ a $52 \%$ do respectivo custo no processo IFAS e, sob alta taxa, de $50 \%$ a $59 \%$.

Como poderia ser esperado, nota-se que à medida que o preço do terreno aumenta, Atibaia menor e São Paulo maior valor, a diferença entre os custos dos respectivos processos reduz. Isto devido ao fato do processo IFAS requerer menor área.

A fim de avaliar a influência do custo de aquisição dos meios suporte, considerou uma redução dos mesmos para R $\$ 900,00$. Com isso, o processo IFAS, de forma geral, se tornou menos desvantajoso e até alcançou valores relativos ao custo final de implantação igual ao processo lodo ativado alta taxa, quando considerado a população de 500.000 habitantes, conforme apresenta a Tabela 7 a seguir.
Em relação ao consumo de energia elétrica, conforme mencionado, pode-se estimar o custo de operação considerando a tarifa de consumo no horário de ponta (horário de ponta seca) de $\mathrm{R} \$ 1,00106 /$ kW.h, a tarifa de consumo no horário fora ponta (horário fora de ponta seca) igual a $\mathrm{R} \$ 0,15912$ / kW.h e o valor de demanda faturável de $\mathrm{R} \$ 7,99 /$ $\mathrm{kW}$, que representa o preço praticado pela concessionária de energia elétrica do Estado São Paulo (AES/Eletropaulo). Apresentam-se assim, segundo modelo de cálculo proposto COSTA \& SILVA na Tabela 8 , as estimativas de custos com energia elétrica para a aeração durante 20 anos de operação dos sistemas de tratamento. Estes custos foram trazidos a valores presentes segundo taxa de juro anual estimada em 10\%. Trata-se apenas de uma aproximação inicial de uma situação que envolve incertezas relativas ao mercado financeiro e à política tarifária de energia elétrica.

Como seria esperado, para os processos trabalhando sob alta taxa os custos relativos ao consumo de energia elétrica para a aeração resul-

Tabela 5 - Custos finais para implantação $(R \$)$ dos processos em cada uma das cidades e para população de 50.000 hab.

\begin{tabular}{c|c|c|c|c|c|}
\multirow{2}{*}{ Processos } & \multicolumn{5}{|c}{ Cidades } \\
\cline { 2 - 6 } & Atibaia & Vinhedo & Jundiaí & Valinhos & São Paulo \\
\hline $\begin{array}{c}\text { Lodo ativado com } \\
\text { aeração prolongada }\end{array}$ & 1.388 .165 & 1.442 .725 & 1.470 .005 & 1.510 .925 & 1.811 .005 \\
\hline IFAS(*) $^{*}$ & 3.819 .128 & 3.853 .768 & 3.871 .088 & 3.897 .068 & 4.087 .588 \\
\hline $\mathrm{R}(\%)$ & 36 & 37 & 38 & 39 & 44 \\
\hline
\end{tabular}

(*) IFAS com A/M de 0,15 kgDBO/kgSSV.d 


\begin{tabular}{c|c|c|c|c|c|}
\multirow{2}{*}{ Processos } & \multicolumn{5}{|c|}{ Cidades } \\
\cline { 2 - 6 } & Atibaia & Vinhedo & Jundiaí & Valinhos & São Paulo \\
\hline $\begin{array}{c}\text { Lodo ativado } \\
\text { alta taxa }\end{array}$ & 3.698 .987 & 3.803 .987 & 3.856 .487 & 3.935 .237 & 4.512 .737 \\
\hline IFAS(1) & 7.330 .564 & 7.368 .004 & 7.386 .724 & 7.414 .804 & 7.620 .724 \\
\hline $\mathrm{R}(\%)$ & $50 \%$ & $52 \%$ & $52 \%$ & $53 \%$ & $59 \%$ \\
\hline $\begin{array}{c}\text { Lodo ativado } \\
\text { convencional }\end{array}$ & 4.308 .904 & 4.434 .904 & 4.497 .904 & 4.592 .404 & 5.285 .404 \\
\hline IFAS(2) & 9.803 .765 & 9.855 .405 & 9.881 .225 & 9.919 .955 & 10.203 .975 \\
\hline R (\%) & $44 \%$ & $45 \%$ & $46 \%$ & $46 \%$ & $52 \%$
\end{tabular}

(1) IFAS com A/M de 0,6 kgDBO/kgSSV.d

(2) IFAS com A/M de $0,4 \mathrm{kgDBO} / \mathrm{kgSSV} . \mathrm{d}$

Tabela 6- Custos finais para implantação $(R \$)$ dos processos em cada uma das cidades e para população de 500.000 hab.

\begin{tabular}{c|c|c|c|c|c}
\multirow{2}{*}{ Processos } & \multicolumn{5}{|c}{ Cidades } \\
\cline { 2 - 6 } & Atibaia & Vinhedo & Jundiaí & Valinhos & São Paulo \\
\hline $\begin{array}{c}\text { Lodo ativado com } \\
\text { aeração prolongada }\end{array}$ & 1.388 .165 & 1.442 .725 & 1.470 .005 & 1.510 .925 & 1.811 .005 \\
\hline IFAS(1) & 2.196 .078 & 2.230 .718 & 2.248 .038 & 2.274 .018 & 2.464 .538 \\
\hline $\mathrm{R}(\%)$ & 63 & 65 & 65 & 66 & 73 \\
\hline $\begin{array}{c}\text { Lodo ativado } \\
\text { alta taxa }\end{array}$ & 3.698 .987 & 3.803 .987 & 3.856 .487 & 3.935 .237 & 4.512 .737 \\
\hline IFAS(2) & 4.231 .290 & 4.268 .730 & 4.287 .450 & 4.315 .530 & 4.521 .450 \\
\hline $\mathrm{R}(\%)$ & 87 & 89 & 90 & 91 & 100 \\
\hline $\begin{array}{c}\text { Lodo ativado } \\
\text { convencional }\end{array}$ & 4.308 .904 & 4.434 .904 & 4.497 .904 & 4.592 .404 & 5.285 .404 \\
\hline IFAS(3) & 5.543 .465 & 5.595 .105 & 5.620 .925 & 5.659 .655 & 5.943 .675 \\
\hline $\mathrm{R}(\%)$ & 78 & 79 & 80 & 81 & 89
\end{tabular}

(1) IFAS com A/M de 0,15 kgDBO/kgSSV.d (2) IFAS com A/M de 0,6 kgDBO/kgSSV.d (3) IFAS com A/M de 0,4 kgDBO/kgSSV.d

Tabela 7- Custos finais para implantação $(R \$)$ dos processos em cada uma das cidades (meios suporte igual a $R \$ 900,00$ )

\begin{tabular}{c|c|c|c|c} 
População (hab.) & $\begin{array}{c}\text { Processo de } \\
\text { Tratamento }\end{array}$ & $\begin{array}{c}\text { Potência } \\
(\mathrm{kW} . \mathrm{h} / \mathrm{h})\end{array}$ & $\begin{array}{c}\text { Custo em } \\
20 \text { anos (R\$) }\end{array}$ & $\begin{array}{c}\text { Custo presente } \\
-20 \text { anos (R\$) }\end{array}$ \\
\hline \multirow{3}{*}{500.000} & $\begin{array}{c}\text { Lodo ativado } \\
\text { alta taxa }\end{array}$ & 933 & 42.884 .946 & 6.374 .574 \\
\cline { 2 - 5 } & IFAS & 933 & 42.884 .946 & 6.374 .574 \\
\cline { 2 - 5 } & R (\%) & - & - & - \\
\hline \multirow{5}{*}{500.000} & $\begin{array}{c}\text { Lodo ativado } \\
\text { convencional }\end{array}$ & 1.252 & 57.717 .333 & 8.579 .314 \\
\cline { 2 - 5 } & IFAS & 1.672 & 76.999 .436 & 11.445 .476 \\
\cline { 2 - 5 } & R (\%) & - & 75 & 75 \\
\hline \multirow{3}{*}{50.000} & $\begin{array}{c}\text { Lodo ativado } \\
\text { aeração prolongada }\end{array}$ & 221 & 10.189 .205 & 1.514 .560 \\
\cline { 2 - 5 } & IFAS & 298 & 13.736 .080 & 2.041 .781 \\
\cline { 2 - 5 } & R (\%) & - & 74 & 74 \\
\hline
\end{tabular}

Tabela 8- Estimativa dos custos de energia elétrica 
taram idênticos, devido ao fato de terem sido considerados os mesmos valores de necessidade de oxigênio e de concentração de oxigênio dissolvido nos tanques de aeração. No entanto, para as variantes convencional e aeração prolongada, o processo de lodo ativado apresentou menor custo devido ao consumo de energia elétrica para aeração, em torno de $74 \%$ a $75 \%$ do custo do processo IFAS.

Deve ser lembrado que estas diferenças percentuais são relativas apenas à construção e operação dos tanques de aeração. Embora possa ser considerado que, de acordo com os procedimentos de cálculo utilizados neste estudo, os custos das demais unidades que compõem a ETE serão os mesmos, as diferenças percentuais entre os custos dos processos de lodo ativado e IFAS reduzem-se significativamente em relação àquelas obtidas considerando-se apenas os tanques de aeração.

\section{Conclusão}

Sob as condições impostas nesta simulação, foi possível concluir, de maneira geral, que o processo de lodo ativado é mais econômico do que o IFAS em relação à implantação dos reatores e aos gastos com energia elétrica para aeração. O principal fator econômico que levou a essa conclusão foi o de que o custo elevado do meio suporte não compensa a economia gerada pela redução da área necessária para a implantação dos reatores. Assim, por meio das hipóteses aqui assumidas, o interesse pelo processo IFAS é maior em situações de implantação ou ampliação de ETE em que não há terreno disponível com área suficiente para o processo de lodo ativado.

\section{Referências bibliográficas}

Associação Brasileira de Normas Técnicas (ABNT) - NBR-12.209. (2012). Elaboração de Projetos Hidráulico-sanitários de Sistemas de Tratamento de Esgotos Sanitários.

COSTA J. M., SILVA L. C. Energia Elétrica - Tarifação. Disponível em: <www.agais.com/eletrica.htm>. Acesso em 23/09/2011 às 10:23hs.

HEM L. J., RUSTEN B., ØDEGAARD H. (1994). Nitrification in a Moving Bed Biofilm Reactor. Water Research, n. 28, v. 6, p. 1425-1433.

JORDÃO E. P., PESSOA C. A. (2009). Tratamento de Esgotos Domésticos. $5^{\mathrm{a}}$ ed. ABES-RJ, 941p.

METCALF \& EDDY. (2003). Inc. Wastewater engineering: treatment and reuse. $4^{\mathrm{a}}$ ed. New York:
McGraw-Hill, 1848p.

MINEGATTI D. V. O. (2008). Caracterização dos Parâmetros de Controle e Avaliação de Desempenho de um Reator Biológico com Leito Móvel (MBBR). Dissertação de mestrado. Departamento de Engenharia Civil - Universidade Federal do Rio de Janeiro, 91p.

ØDEGAARD H., RUSTEN B., WESTRUM T. (1994). A new moving bed biofilm reactor - application and results. Water Science and Tecnhology, n. 29, v. 1011, p. 157-165.

ØDEGAARD H., RUSTEN B., BADIN H. (1993). Small wasterwater treatment plants based on moving bed biofilm reactor. Water Science and Tecnhology, $\mathrm{n}$. 28 , v. 10, p. 351-359.

RUSTEN B., MCCOY M., PROCTOR R., SILJUDALEN J.G. (1998). The innovative moving bed biofilm reactor/Solids contact reaeration process for secondary treatment of municipal wastewater. Water Environment Research, n. 70, v. 5, p. 1083-1089.

RUSTEN, B; HEM, L; ØDEGAARD, H. (1995). Nitrification of municipal wastewater in moving bed biofilm reactor. Water Environment Research, Vol 67 (1), pp 75-86.

RUSTEN B., SILJUDALEN J. G., NORDEIDET B. (1994). Upgrading to nitrogen removal with the KMT moving bed biofilm process. Water Science and Technology, n. 29, v. 12, p. 185-195. 